\title{
METODOLOGÍA PARA EL ESTUDIO \\ DE LAS MUJERES \\ Y LA SOCIEDAD RURAL
}

\author{
POR \\ MIREIA BAYLINA FERRÉ*
}

\section{Introducción}

Los estudios de geografía y género se han asociado desde sus inicios a la metodología y los métodos cualitativos de investigación. Aunque es cierto que éstos métodos encajan con los objetivos de la investigación feminista, con muchos de los temas de análisis, y que al principio algunas investigadoras feministas tendieron a rechazar los métodos cuantitativos por su conexión con el positivismo, la metodología y los métodos cuantitativos también han sido y son utilizados. De hecho, los primeros trabajos de género obtuvieron reconocimiento mostrando que los análisis cuantitativos que no consideraban el género no sólo eran injustos sino que podían conducir a conclusiones equivocadas. Los estudios que «contaban a las mujeres» fueron muy importantes y han demostrado el valor de los métodos cuantitativos en «hacer visible lo invisible» (Mattingly \& Falconer Al-Hindi, 1995; McLafferty, 1995). Sin embargo durante las pasadas décadas se ha asistido a un prolífico debate sobre este dualismo entre metodología cuantitativa y cualitativa, subrayando que ambas son exclusivas ya que provienen de distintas posiciones epistemológicas. Recientemente, un número creciente de investigadores/as han argumentado que esta perspectiva dicotómica es demasiado simple y puede ser contraproducente, y los nuevos discursos han conducido al desarrollo de estrategias de

* Mireia Baylina Ferré. Dpto. de Geografía, Universidad Autónoma de Barcelona.

Estudios Geográficos, LXV, 254, 2004 
investigación que reconocen la complementariedad de algunas técnicas cuantitativas y cualitativas.

Por otra parte, los avances teóricos en geografía feminista, en las epistemologías post-positivistas y los debates sobre metodología a lo largo de estos últimos años han consolidado la metodología cualitativa, no sólo en los estudios de género sino en la geografía en general, y han llevado a la práctica un pluralismo metodológico. Esta idea se constata también para los estudios sobre género y geografía rural ${ }^{1}$.

Las geógrafas ruralistas que han tratado temas de género han prestado desde los inicios mucho interés por las cuestiones metodológicas. Entre los primeros estudios sobre mujeres, género y medio rural destacan los que tratan sobre el desarrollo y los países del Tercer Mundo. La dificultad para obtener información estadística para las investigaciones y unos objetivos de investigación más amplios que buscaban el conocimiento en profundidad del trabajo y de la vida de las mujeres y las posibilidades de mejora de su situación, hicieron necesaria la reflexión metodológica previa. Lourdes Benería, en la edición de Women and Development. The sexual division of labour in rural societies, 1982, recoge algunos ejemplos interesantes que tratan sobre el trabajo productivo y reproductivo de las mujeres rurales en los países en desarrollo, de los que deduce la necesidad de redefinir los conceptos que están detrás de las estadísticas y que contribuyen a omitir o a subestimar la contribución de las mujeres al trabajo. (La investigación está financiada por el Programa sobre Mujeres Rurales de la Organización Internacional del Trabajo). Janet Momsen y Vivian Kinnaird (1993) tratan la relación entre género y desarrollo en África, Asia y América Latina a través de la perspectiva de las geógrafas africanas, asiáticas, latinoamericanas y occidentales. El mensaje de las autoras se dirige en muy buena parte a la metodología, destacando que los datos estadísticos generales que ofrecen las Naciones Unidas ofrecen insuficiente evidencia para conceptuar la respuesta de las mujeres a las nuevas situaciones de crisis del medio rural de sus países: cuestiones relacionadas a las diferencias de salud por razones de género, temas de gestión de recursos, in-

\footnotetext{
${ }^{1}$ Algunos indicadores que revelan la creciente aceptación de los métodos de investigación cualitativa son su enseñanza en cursos de distintos niveles; su aceptación en proyectos de investigación financiados; la presencia de revistas científicas y grupos de investigación especializados en esta metodología; y la publicación de investigaciones basadas - o acerca de- los métodos cualitativos en las revistas científicas de ciencias sociales de más prestigio (Raghuram, Madge \& Skelton, 1998; Crang, 2002; Rose, 2002).
}

Estudios Geográficos, LXV, 254, 2004 
teracción rural/urbana, interacción rural/rural a través de la migración, relación entre los roles productivo y reproductivo y la toma de decisiones a nivel familiar, etc. En definitiva, demuestran que para conocer los porqué de la sociedad rural del Tercer Mundo se necesita investigación empírica desde la base. Más recientemente, en el libro editado por John Paul Jones, Heidi Nast y Susan Roberts (1997), Thresholds in feninist geography: difference, methodology and representation, una de las autoras Vidyamali Samarasinghe (curiosamente contribuidora también en el libro citado de Benería) expone el método de análisis espacio-temporal para comprender a las sociedades rurales agrícolas del Global South ${ }^{2}$. Samarasinghe puntualiza que la dimensión olvidada del trabajo de las mujeres en la unidad familiar no es exclusivo de las sociedades en desarrollo y que el no reconocimiento de las actividades reproductivas como trabajo es una cuestión que afecta a las mujeres de todo el mundo. Sin embargo, en el Sur Global y en la producción de autosubsistencia, el no reconocimiento asume una dimensión más amplia debido a la naturaleza del trabajo de las mujeres en estas sociedades. Los datos recogidos agregados a nivel nacional no han reflejado la contribución de las mujeres a la supervivencia económica debido al uso continuado de una metodología que se basa en conceptualizaciones equivocadas sobre el «trabajo». Quince años después de su capítulo en Women and Development (Benería, 1982), la autora reconoce el interés por las Naciones Unidas, la Organización Internacional del Trabajo y el Banco Mundial por el sub-empleo de las mujeres pero critica que no han afrontado el significado del término «trabajo» para abordar el problema a fondo (Samarasinghe, 1996). Con estos ejemplos queremos reconocer la contribución, sobre todo en los inicios, de las geógrafas rurales a la metodología en otras partes del mundo, que no se recogen en las páginas siguientes.

En este artículo se desarrolla, en primer lugar, la importancia de la teoría feminista para el estudio de la mujer rural; a continuación se trata la relación entre los temas de estudio, los paradigmas y las metodologías utilizadas en la evolución de los estudios sobre la mujer rural a nivel nacional e internacional (predominantemente en Europa); y finalmente se valoran las posibilidades, dificultades y la adecuación de los métodos cualitativos y cuantitativos para estas investigaciones.

${ }^{2}$ Término que prefiere la autora en lugar de «Tercer Mundo».

\section{$-7-$}

Estudios Geográficos, LXV, 254, 2004 
El artículo trata dos categorías complejas de análisis: mujer y medio rural. Sin entrar a debatir en profundidad el significado de estos términos, es conveniente cómo van a ser considerados en adelante. Se trata la categoría mujer en el sentido biológico y cultural, a fin de dar cabida al sexo como una construcción cultural, y como un concepto plural para considerar a las mujeres como un grupo heterogéneo. Es evidente que existen diferencias entre las mujeres, lo que hay que reconocer es el poder de una determinada versión de la feminidad en la sociedad rural y discutir su exclusividad en este entorno. Por su parte, cuando hablamos de la mujer rural nos referimos a la mujer que reside en el medio rural. Finalmente, el medio rural se concibe en un sentido amplio a fin de incluir las distintas dimensiones, características, significados y representaciones de lo rural en distintos contextos. Quizás el significado común al medio rural actual de las sociedades avanzadas es la noción de diversidad y fragmentación, resultado de los cambios económicos, sociales y medioambientales que han experimentado las áreas rurales desde inicios de los años ochenta (Marsden, 1998). Los espacios rurales se han desarrollado de forma desigual en los últimos años debido a fuerzas regulatorias externas e internas (cada región está vinculada de forma distinta a la economía regional e internacional, así como a formas de regulación nacional) (Lowe et al., 1993), y la diferenciación rural se expresa en el espacio de forma distinta. Así pues, la lectura de «lo rural» en este artículo es la de un medio dinámico, complejo y diferenciado, y la metolodogía para su estudio (y en concreto, para el estudio de la sociedad rural) es transversal a esta concepción.

\section{Relevancia de la teoría feminista en la investigación} sobre la mujer y la sociedad rural

La epistemología feminista es básica para el estudio de la sociedad rural ya que considera cómo el género influye en lo que se considera conocimiento, y cómo los roles y relaciones de género, socialmente construidos, influyen en la producción de este conocimiento. El género condiciona cómo las personas experimentan el mundo, cómo interactúan con otros y qué oportunidades o privilegios se les ofrecen o niegan. En este sentido, uno de los elementos más importantes en las relaciones de género es cómo se solidifican relaciones de poder en

Estudios Geográficos, LXV, 254, 2004

$$
-8-
$$


una sociedad a través de la opresión (violencia, discriminación, marginalización) y del privilegio (poder sobre los otros). En los procesos de producción de conocimiento los oprimidos y los privilegiados tienen distintos roles. Históricamente las mujeres se han contemplado como menos significativas en la producción de lo qué cuenta como conocimiento (Cope, 2002). Por ejemplo, en los análisis sobre el mercado laboral, una investigadora feminista querrá saber, además de dónde se encuentran los puestos de trabajo o cuáles son las cualificaciones de los trabajadores, etc., dónde se concentran las mujeres y de dónde se excluyen, si ganan lo mismo que los hombres por el mismo tipo de trabajo, cómo afectan las responsabilidades domésticas en el trabajo remunerado?, etc. Si se ignoran estas cuestiones, el estudio resulta incompleto y parcial, ya que asume que hombres y mujeres tienen un comportamiento igual, y además reconoce que las relaciones de género no tienen ningún impacto en la ocupación.

Así pues, una epistemología feminista toma el género como central para la producción de conocimiento y reconoce su influencia en todo el proceso de investigación. El género es relevante desde la formulación de preguntas hasta la representación de los resultados de la investigación. Las investigadoras feministas plantean preguntas de género desde el inicio (por ejemplo en una investigación sobre cómo las personas utilizan una plaza (en un pueblo, por ejemplo) preguntarnos quien y para $q u e ́$ la utiliza en términos de género, clase, edad, etnicidad, sexo, etc., y en este sentido se cuestiona cómo las relaciones de poder como el género y otras pueden establecer diferencias en la utilitzación de la plaza); en la recogida de información se preocupan de qué datos cuentan para ellas (aquí se incluyen una variedad de fuentes como historias orales, cartas, diarios, fotografías, cine, canciones, paisajes, etc.) y de cómo se obtienen estos datos (la forma de obtención de información, la relación entre investigador e investigado, el lugar donde se desarrolla el proceso, etc., son elementos fundamentales para la construcción del proyecto de investigación). El género también influye en la elección del método de análisis. En principio, una epistemología feminista no requiere un método específico, sino métodos adecuados al objeto de estudio, y por lo tanto, consistentes con los objetivos feministas. Por ejemplo, si el objetivo es comprender las implicaciones sociales y económicas de las mujeres como agricultoras de subsistencia, los datos estadísticos «oficiales» serán poco útiles para conocer lo que la observación es-

Estudios Geográficos, LXV, 254, 2004 
pontánea sugiere como realidad. No obstante, la constatación del déficit de información gubernamental puede estimular otro proyecto de investigación relacionado con las deficiencias metodológicas para analizar el género. Igualmente, si se pretende analizar una cadena de valor global en la que parte de la producción se realiza de manera informal (por lo tanto, sin ningún dato estadístico), si no se recurre a los métodos cualitativos para acceder a los trabajadores/as y conocer el trabajo no se obtiene ninguna información sobre una parte muy importante del proceso (Rowbotham \& Tate, 1998; Carr, Chen \& Tate, 2000). Por su parte, el género interviene en la interpretación de los resultados, y en este sentido el «conocimiento situado» (Haraway, 1988) ${ }^{3}$ es muy útil para reconocer el contexto del investigador, los sujetos y el lugar (social y físico) en el análisis de cómo el género influye en la producción de conocimiento. Finalmente, en la representación de los resultados la perspectiva de género incide en la búsqueda de fórmulas diversas para romper la tradicional dinámica de poder entre el investigador y el investigado (por ejemplo, la discusión de los resultados con los investigados o la co-autoría de los mismos (Townsend, 1995, 1999), la coproducción de la investigación, la investigación colaborativa (Monk et al., 2002; Manning et al., 2002), o la exposición de resultados en foros diversos que puedan tener una influencia más directa en la personas (informes para políticos, prensa, tv, etc.).

Por su parte, la geografía feminista sostiene que los hombres y las mujeres estan situados de un modo distinto en el mundo y que su relación con los lugares donde viven también es diferente. Y estas diferencias son el resultado de las desigualdades que sitúan a la mujer en un puesto inferior al del hombre, donde sufre la opresión en distintos espacios y tiempos (McDowell, 2000). Los estudios en esta línea, por bien que se inician en paralelo con la aparición de las teorías feministas, se toleran y aceptan muy tarde en el mundo de la geografía, sobre todo en nuestro país (Garcia Ramon, 1989a; Sabaté \& Tulla, 1992), pero también a nivel internacional (McDowell, 2000). En relación a la geografía rural los estudios desde esta perspectiva son todavía más recientes. A nivel internacional este hecho puede deberse a una in-

${ }^{3}$ Entendido como la localización en un espacio cultural que configura y modela la visión del mundo de cada persona. Se trata de hablar de un lugar y desde un lugar, tomando partido de las cosas, afrontando con responsabilidad las interpretaciones (Haraway, 1988).

Estudios Geográficos, LXV, 254, 2004

$$
-10-
$$


corporación más lenta de las nuevas corrientes teóricas por parte de los geógrafos/as rurales, y a que los estudios desde la perspectiva de género vienen más de las geógrafas feministas que de las ruralistas (Little, 2001); y a nivel español, el retraso en comparación con otros países europeos puede explicarse por el androcentrismo que ha caracterizado a los estudios rurales en nuestro país y al menor y más lento desarrollo de los estudios de género en gran parte de las ciencias sociales (Garcia Ramon \& Baylina, 2000).

\section{Estudios rurales, género y metodología}

Los estudios rurales desde una perspectiva de género se inician en Europa en la década de los setenta y su objetivo principal es hacer visibles a las mujeres, una decisión política que comparten todas las geógrafas feministas a fin de corregir la ausencia de las mujeres como sujetos de investigación geográfica (Hanson \& Monk, 1982; WGSG, 1984; Garcia Ramon et al., 1988; Sabaté, 1989; Bowlby et al., 1989; Bowlby, 1992). Las geógrafas señalan la relevancia para las mujeres de muchos temas analizados desde una orientación masculina e investigan sobre temas que preocupan a las mujeres. Un trabajo que abre y legitima nuevas áreas de investigación geográfica y pone de relieve la naturaleza androcéntrica de la geografía tradicional (Little, 2001).

Los estudios rurales de esta época sitúan el interés en la contribución de las mujeres en las explotaciones familiares agrarias y se documentan todas las actividades que realizan destacando su centralidad dentro de la explotación. Estos estudios contribuyen a la comprensión de la vida diaria de las mujeres, incrementa el conocimiento sobre las necesidades y problemas en las comunidades rurales y suponen una apertura de nuevos temas y nuevas escalas de análisis en geografía rural, como la de la unidad familiar. También ponen de relieve la interconexión entre lo público y lo privado, la producción y la reproducción. En este sentido, se reexaminan conceptos clave como el de trabajo y el de familia, que hasta entonces ignoraban la presencia y contribución de las mujeres en las explotaciones agrarias europeas (Whatmore, 1994). El trabajo porque se identificaba con las actividades agrícolas y se medía de acuerdo con las categorías que se basaban en el modelo masculino (por ejemplo, una jornada laboral estándar masculina), eclipsando el resto de trabajo

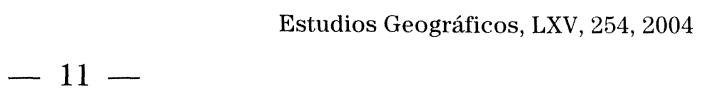


realizado en la unidad familiar. Y la familia porque se trataba como una entidad orgánica representada por el cabeza de familia masculino, oscureciendo de este modo las divisiones sociales y las desigualdades dentro de la familia (Garcia Ramon \& Baylina, 2000). En España, los primeros estudios (mediados de los años ochenta) también se centran en el intento de inventariar y contabilizar el trabajo de la mujer en la agricultura, tras descubrir su invisibilidad e infravaloración.

Desde el punto de vista metodológico, una de las primeras cuestiones que se plantean las investigaciones es saber si existen fuentes de datos realmente útiles, detectando muy pronto la insuficiencia de datos estadísticos para conocer el trabajo de la mujer, tanto en la esfera productiva como en la reproductiva. La falta de unas estadísticas precisas va unido a las cuestiones conceptuales (por ejemplo, no se reúnen datos sobre actividades no remuneradas porque no se califican como trabajo, ni se contabilizan las actividades remuneradas desarrolladas de forma informal). La constatación de esta insuficiencia es también objeto de estudio en análisis detallados sobre las posibilidades de las fuentes estadísticas (Garcia Ramon, 1989b; Momsen, 1989; Solsona, 1989; Sabaté, 1989). En general, en esta primera etapa de análisis de los roles de género, se utiliza mayoritariamente una metodología cualitativa. Esto es más real para Europa que para España, dónde se realizan algunos estudios importantes (particularmente desde la sociología rural) sobre la situación socioeconómica de la mujer rural a partir de métodos estadísticos (Camarero et al., 1991; Vicente-Mazariegos et al., 1991-94; Sampedro, 1996). La metodología cualitativa, quizás más desarrollada por las geógrafas (Sabaté, 1989; Garcia Ramon et al., 1995), se generaliza más tarde entre todos los ruralistas, y de hecho, la evolución de los estudios de geografía y género han contribuido a extender el uso de la misma, en España, y ampliamente en Europa occidental (Crang, 2002; Rose, 2002).

El estudio de la posición de las mujeres en la agricultura aporta un logro teórico de gran importancia: el desarrollo del concepto de género como una división social construida culturalmente. Este hecho provoca el paso de la investigación centrada en cómo la mujer encaja en el panorama de una explotación agraria definida por las experiencias y perspectivas de los hombres, a la investigación que describe la vida en la explotación y en la comunidad rural dónde las experiencias y las ambiciones de las mujeres son constitutivas de este contexto (Whatmore, 1994).

Estudios Geográficos, LXV, 254, 2004

$$
-12-
$$


Muy pronto el foco de las investigaciones se sitúa en la explicación de las desigualdades y ello proporciona más estímulos para los estudios de género en geografía rural, entre ellos, las divisiones de trabajo dentro de la familia y de la sociedad rural o la pluriactividad y el trabajo fuera de la explotación. Estos temas dan pie a un gran número de investigaciones que tienen por objetivo la comprensión del proceso de trabajo familiar como un proceso generizado, no sólo por la distribución de tareas sino también por las condiciones sociales que existen detrás del trabajo de hombres y de mujeres. El estudio de las diferencias en la posición de hombres y mujeres en las explotaciones familiares y en el mercado laboral en general se analizan en términos de la negociación diaria de la autoridad y la influencia en las decisiones de la unidad familiar o del lugar de trabajo. Esta teoría más general de las relaciones de género permite descubrir que las posiciones se encuentran en el contexto de valores y prácticas institucionalizadas que empoderan a los hombres y desempoderan a las mujeres como agentes sociales.

El cambio de énfasis desde las mujeres a las relaciones de género da lugar a un trabajo teórico más complejo sobre la variedad en la construcción espacial del género y las formas específicas por las cuales la masculinidad y la feminidad varían entre espacios, y también entre clases, razas, edad, identificación sexual y cultural (England, 1994; Kobayashi, 1994). La atención a los diversos lugares y a las diversas experiencias de las mujeres establece una nueva relación entre teoría y metodología. El objetivo es evitar las generalizaciones sobre las experiencias de las mujeres para entender su especificidad en tiempos particulares y en diferentes lugares y hacerlo desde un conocimiento situado (Hanson, 1992). Por su parte, la escala de análisis local y del hogar se enfatiza en esta nueva etapa, con lo cual los nuevos avances teóricos reclaman una mayor atención a los métodos etnográficos de investigación. Precisamente estos métodos que dan voz a las personas, permiten, además de caracterizar su experiencia diaria, identificar nuevas categorías de trabajo, ya que no se trata de adecuar lo que hacen las mujeres en unas categorías convencionales impuestas, sino de crear otras nuevas a partir de su realidad. Por lo tanto, pueden contribuir a cambiar la legislación (sobre pensiones, subsidios por desempleo o enfermedad) y a otorgarles más derechos.

De hecho los esfuerzos teóricos realizados en el marco de la teoría de los roles y de las relaciones de género apoyados con una metodología que

Estudios Geográficos, LXV, 254, 2004 
reconoce las relaciones sociales de la investigación y tiene objetivos emancipatorios para todos/as los que participan del proceso, y con métodos adecuados como las entrevistas en profundidad, la observación participante, los grupos de discusión, las historias de vida, o los análisis de documentos, han contribuido a repensar la naturaleza de la explotación familiar agraria, que ha sido la base de la mayor parte de los estudios rurales desde la perspectiva de género, sobre todo en sus inicios (Whatmore, 1994). En este sentido, ya no se contempla la familia como una entidad universal y se reconoce la diversidad de formas de familia en diferentes lugares y tiempos; algo básico para comprender el comportamiento de los y las jóvenes rurales actuales y estudiar la viabilidad futura de las explotaciones familiares, las culturas y los entornos rurales.

Los años noventa introducen cambios importantes en los estudios rurales inducidos por el postmodernismo, la evolución del movimiento feminista y la evolución de la geografía agraria en el marco más general de la geografía rural, más interesada por la vertiente teórica y analítica de la geografía (Whatmore, 1993; Marsden, 1994, 1996; Little, 1999). En general el énfasis recae en la noción de la diferencia, no sólo para comprender las experiencias de las mujeres sino para cambiar las asunciones culturales que envuelven las relaciones entre la masculinidad y la feminidad (Little, 2001). Ello lleva a nuevos centros de interés como la «otredad», la construcción de la identidad y del paisaje y el énfasis en la espacialidad de la naturaleza (Cloke, 1997; Murdoch y Pratt, 1993). Se estudian grupos olvidados de mujeres y hombres en investigación como los/as jóvenes, discapacitados/as, mayores, niños/as... (Gidarakou, 1999; Matthews et al., 2000; Panelli, 2001), se cuestiona los conceptos de hombre, mujer, mujer rural y de género como categoría de análisis (o más que nada se cuestiona su centralidad permanente y su exclusividad en una investigación feminista, para dar cabida a la etnicidad, edad, discapacidad, etc.) (Little, 2001; Johnson, 2002). La incertidumbre que envuelve la investigación feminista es una oportunidad, pues, para deconstruir estas categorías y para poner el énfasis en la noción de identidad de género, es decir, cómo las personas experimentan el género y la sexualidad y cómo éstos son discutidos. En este sentido, se empieza a reconocer el significado de las representaciones diarias de lo que quiere decir ser un hombre o una mujer, que es lo que da sentido y legitimidad a las relaciones de poder en la unidad familiar, explotación agraria, o en la comunidad local. Lo que importa es ver como las iden-

Estudios Geográficos, LXV, 254, 2004

$$
-14-
$$


tidades y prácticas dominantes de género se acomodan, sustentan, discuten y transforman.

El análisis de las identidades rurales de género por parte de los geógrafos/as ha estado muy influenciado por los debates sobre la construcción cultural y la representación de la ruralidad (Little, 2002). Los trabajos en el Reino Unido y otros países de Europa occidental sobre el idilio rural (Halfacree, 1996; Cloke \& Little, 1997; Hughes, 1997; van Dam, Heins \& Elbersen, 2002) muestran como éste lleva implícito fuertes expectativas en las identidades de los hombres y de las mujeres y de las relaciones de género. Pertenecer a una comunidad rural significa cumplir con unas determinadas nociones de lo que supone ser un hombre o una mujer, y no hacerlo amenaza el sentimiento de aceptación dentro de la sociedad rural.

Los estudios sobre la asociación entre la ruralidad y la identidad de género son todavía incipientes aunque muy prometedores para el futuro. La consideración a la hibridez y la fluidez en la identidad de género permite un análisis más sofisticado de la marginalidad rural y contribuye a establecer más vínculos entre la construcción cultural de la ruralidad y los aspectos de los roles y relaciones de género que son valorados dentro de estas construcciones dominantes. Otros centros de interés que abre o re-abre esta nueva perspectiva son las relaciones entre la naturaleza, paisaje y sexualidad (Woodward, 1998; Monk, 1999); la construcción de la masculinidad y de la feminidad (Woodward, 1998; Rural Sociology, 2000); la (re)construcción de la identidad como mujer rural y la consiguiente (re)construcción de la ruralidad; el uso del espacio rural por parte de hombres y mujeres rurales; el (re)descubrimiento de la contribución de las mujeres a la innovación y al progreso de la agricultura; la naturaleza generizada del poder en el mundo rural y los procesos de exclusión de la mujer (Fincher \& Panelli, 2001; el ecofeminismo y el desarrollo sostenible; o las identidades de los hombres rurales. En relación a éste último tema cabe decir que se ha trabajado todavía poco sobre la vida de los hombres, la relación cambiante entre hombres y mujeres en el medio rural, las expectativas y experiencias de la masculinidad rural en la familia y en la comunidad, o la relación entre la construcción de la masculinidad y la sociedad rural (Little, 1997, 2002). Algunas excepciones notables son las contribuciones de las investigadoras nórdicas (Almas \& Haugen, 1991; Brandth, 1995; Brandth \& Haugen, 2000). El estudio de los hombres y de su identidad es fundamental 
en sí mismo, para el conocimiento de la sociedad rural y también para conocer legítimamente la posición relativa de las mujeres (McDowell, 2000). En este nuevo contexto los investigadores/as examinan las vidas de los distintos residentes rurales, sus identidades particulares y las formas en que estas identidades interactúan con la ruralidad, facilitando o restringiendo el hecho de mostrar su identidad (un buen ejemplo de este tipo de estudios es el libro editado por Paul Cloke y Jo Little, Contested countryside cultures. Otherness, marginalisation and rurality, London, Routledge, 1997) ${ }^{4}$.

Cabe decir que estos trabajos más novedosos coexisten con los estudios más tradicionales sobre los roles de las mujeres, los de la etapa del feminismo empiricista (Harding, 1987) o del «add women and stir». Esto es importante porque demuestra que las perspectivas teóricas en geografía feminista, aunque se presenten de manera secuencial, no se sustituyen ni se excluyen. De hecho, los distintos enfoques pueden y deben coexistir. Aún más, en muchos contextos rurales el hecho de visibilizar a las mujeres es todavía una tarea pendiente, y muy necesaria para avanzar en el cambio social hacia relaciones de género más igualitarias (Garcia Ramon \& Baylina, 2002). Y por otra parte, es positivo que en medio de esta derivación al terreno cultural de la geografía rural de género no se pierda de vista la base política de la geografía feminista ni la estructura material de la desigualdad, que otros enfoques quizás potencian más.

En España, por ejemplo, no se ha avanzado en los estudios sobre identidad ${ }^{5}$, muy desarrollados en los países nórdicos y de la Europa occidental. Pero en cambio se estan desarrollando bajo este enfoque postmoderno estudios muy interesantes sobre género y medioambiente, en particular las movilizaciones de las mujeres en torno a problemas medioambientales (Bru, 1996a; 1996b) y la implicación de las mujeres en la agricultura ecológica como productoras y consumidoras (Sabaté, 2000; López García, 1999, 2000) y se siguen realizando trabajos desde enfoques más tradicionales.

\footnotetext{
${ }^{4}$ En geografía existe una notable producción de estudios sobre la (re)construcción de la historia de la geografía en clave de género a través de los libros de viajes (Domosh, 1991; Albet \& Garcia-Ramon, 1999) o de la autobiografía (Monk, 2001; Buttimer, 2001; Eyles, 2001).

${ }^{5}$ Nos referimos al binomio identidad de género y medio rural, ya que sí existen en geografía feminista como los trabajos relacionados con el discurso colonial y la historia de la geografía (Garcia Ramon \& Nogué, 1999).
}

Estudios Geográficos, LXV, 254, 2004

$$
-16-
$$


En general, este giro cultural en geografía rural, que presta más atención a la diferencia y a los aspectos más experienciales de la vida de las personas, ha alentado la adopción de métodos cualitativos de investigación más allá de las entrevistas en profundidad. En particular la observación participante, los grupos de discusión, el uso de fuentes documentales, fotográficas y cinematográficas, las historias de vida y de forma incipiente, las autobiografías (Little, 2001; Thorsen, 1994; Moss, 2001; Crang, 2002). Sin embargo, la coexistencia de trabajos con enfoques distintos evidencia también el uso de técnicas cuantitativas o la combinación de ambas.

\section{Posibilidades, dificultades y adecuación de los métodos cualitativos y cuantitativos}

Como hemos visto, es evidente que existe una estrecha relación entre los métodos etnográficos y los objetivos feministas de investigación. En los inicios de los 80, las geógrafas Janice Monk y Susan Hanson (1982) ya criticaban que gran parte de la investigación en geografía no recogía las necesidades de las mujeres, ni en los objetivos (la geografía positivista no pretendía el cambio social) ni en la metodología, considerando así la mayor parte de estudios geográficos como «pasivamente sexistas». Las autoras pedían la adopción de métodos no sexistas y sensibles al género. Por su parte, Linda McDowell (1992) añadía que una de las razones para excluir la vida de las mujeres en la investigación geográfica se fundamentaba en razones metodológicas, con lo cual había que reexaminar los métodos convencionales y buscar métodos no sexistas de investigación.

Partiendo de estas consideraciones, una de las principales contribuciones del feminismo en los últimos veinte años ha sido hacer visible la masculinización de la ciencia a través de una revisión crítica de distintos modelos de ciencia desde el positivismo al marxismo o humanismo en relación a la concepción del comportamiento humano y la posibilidad de formular leyes sobre el mismo; las características del conocimiento (explicación frente a comprensión) y el objetivo de la ciencia (nomotética o idiográfica) (Sabaté et al., 1995).

Los resultados han puesto de manifiesto que los principios teóricos y la práctica del feminismo conciben la ciencia como una actividad no neutral, y por lo tanto, comprometida con el cambio de la realidad que 
estudia; y tiene por objetivo construir el conocimiento a partir de la experiencia subjetiva, rechazando la pretendida objetividad de la ciencia y la separación entre sujeto y objeto de investigación. Por lo tanto, de la abundante bibliografía sobre temas metodológicos y geografía feminista ${ }^{6}$ y de la recurrente pregunta de si existe o no un método feminista de investigación puede deducirse que la adopción del método de investigación viene después de elegir la base teórica sólida que permita determinar qué, cómo y para qué se investiga.

Los métodos cuantitativos, asociados al modelo de ciencia positivista, han sido frecuentemente rechazados por las investigadoras feministas: por sus principios de neutralidad, objetividad, por su asociación con las cualidades personales asumidas como masculinas; y porque el tipo de datos que manejan es poco adecuado para los temas de investigación feminista ${ }^{7}$. Sin embargo, las geógrafas feministas y ruralistas los han utilizado (análisis de censos, grandes encuestas, etc.) (Prados, 2000; Majoral \& Sánchez, 2000; López García, 2000). De hecho los métodos cuantitativos se valoran positivamente para mostrar la incidencia de un particular fenómeno (medición); para ofrecer marcos más generales; para forzar la inclusión de un tema en la agenda política; para comprobar e invertir las prácticas androcéntricas y para que sirvan a objetivos feministas; y en algunos casos el supuesto anonimato y distancia entre investigador e investigado puede resultar adecuado para tratar temas sensibles, que resultarían dolorosos en una conversación abierta.

Los métodos cualitativos permiten valorar la subjetividad, la implicación personal, la sensibilidad a los fenómenos complejos y únicos, el conocimiento contextual, la perspectiva individual y la posibilidad de crear un intercambio de experiencias entre investigador e investigado (Sabaté et al., 1995; Marcus, 1995; Hanson \& Pratt, 1995). Permiten abordar más los estudios de casos y a pequeña escala (local, comunidad, unidad familiar, cuerpo) y facilitar la comprensión de los fenómenos. Por ejemplo, la observación participante facilita la integración del investigador en el mundo del investigado y poder compartirlo. La entre-

\footnotetext{
${ }^{6}$ Algunas de las publicaciones más significativas son: The Professional Geographer, 1994, 1995, 2001; Canadian Geographer, 37 (1993); Antipode (1995); Jones, Nast \& Roberts (1997); McDowell (1992; 1997; 2000); Moss (2002); Women and Geography Study Group (1997).

${ }^{7}$ En este sentido, una de las críticas principales es la no contextualización de los datos. Los datos cuantitativos se presentan atomizados, «disembodied»; no reconstruyen la experiencia vivida de una forma global, holística.
}

Estudios Geográficos, LXV, 254, 2004 
vista en profundidad permite explorar las experiencias individuales, los valores, las ambiciones ${ }^{8}$. La interpretación de documentos (fotografía, literatura, pintura, cine, tv..) y del paisaje revela información sobre los roles de género y las relaciones de poder basadas en el género, la clase, la sexualidad, y muestran la identificación de las mujeres con sus identidades (Madge, 1997; Monk, 1999). Las historias de vida permiten traducir el conocimiento vivido en conocimiento académico, siendo muy útiles para comprender, por ejemplo, las trayectorias profesionales de las mujeres (Monk, 2001). Y los grupos de discusión, por ejemplo, permiten explorar las experiencias colectivas, dan oportunidad para la discusión y la reflexión, y se reconocen como una técnica que facilita el empoderamiento de los participantes y que puede contribuir al cambio político y social (Pini, 2002). Sin embargo, el uso de métodos cualitativos también genera problemas que tienen que ver con el rigor científico y la ética en el comportamiento del investigador. En el primer caso, el error de utilizar información procedente de pocos casos y pretender generalizar ${ }^{9}$; o la falta de rigor en el control de los métodos para validar la investigación ${ }^{10}$. En el segundo caso, se plantean problemas éticos relacionados con las relaciones de poder que se establecen entre investigador e investigado y durante el proceso de investigación.

Las relaciones de poder existen de forma inevitable y producen determinados efectos en la investigación; por lo tanto su reconocimiento debe permitirnos atenuar sus efectos o reconvertirlas en relaciones más igualitarias. En este sentido se han intensificado los métodos que fomentan la colaboración de las personas investigadas y las implican más directamente, que crean vínculos de solidaridad y permiten luchar contra los privilegios (McDowell, 1988, 1992; Townsend et al., 1994; Esim, 1997; Baylina, 1997; Prats, 1998; Monk et al., 2002).

La posición del investigador (género, clase, edad, etnicidad, sexualidad...) no es solo en términos de poder, sino en cómo nos dirigimos al

${ }^{8}$ Algunas autoras han calificado esta técnica como un «viaje formativo» sobre la vida de las mujeres rurales (Thorsen, 1994).

${ }^{9}$ La generalización de los resultados no se encuentra en los objetivos de la investigación cualitativa. El objetivo en todo caso sería alcanzar una generalización teórica, es decir, que el resultado del estudio de casos contribuyera al desarrollo de teoría (Rose, 2002).

${ }_{10}$ Por ejemplo, es importante insistir en que la transparencia en todos los aspectos del proceso de investigación es un criterio clave para maximizar la credibilidad y la confianza en los resultados.

Estudios Geográficos, LXV, 254, 2004 
investigado y en cómo interpretamos los resultados (WGSG, 1997). Por ejemplo, Jo Little (2001) explica lo útil que fue para ella entrevistar a mujeres rurales británicas, blancas, de clase media dado que ella se ajusta a estas características, vivió en el campo, le gusta ir al campo, etc. Sin embargo, tuvo problemas cuando quiso comprobar su grado de «otredad» respecto a sus entrevistadas en relación a la ideología doméstica. Lo que para ella era lo «adecuado» en su rol de género, no lo era para las demás, y cualquier sugerencia a la situación de desventaja o de marginalidad impuesta por las restricciones derivadas de su rol doméstico para muchas fue ofensivo o inadecuado. Little no pudo construir una «alianza de investigación» entre todas ellas.

Descubriendo estos temas de posicionalidad y reflexividad ayuda a aumentar la sensibilidad en el proceso de investigación y minimizar las relaciones de poder. En cualquier caso lo importante no es tanto que las posiciones subjetivas del investigador e investigado sean idénticas, sinó que las estrategias para la recogida de datos y para la difusión de la investigación sean acordes a las características, experiencias y necesidades de la persona que se investiga.

Los nuevos enfoques que enfatizan la diferencia han introducido nuevos problemas metodológicos: por ejemplo el caso de mujeres blancas que estudian mujeres negras; mujeres del primer mundo que estudian mujeres del tercer mundo (Kim, 1997); mujeres que investigan a hombres y la ética de utilizar la feminidad para extraer información de un informante masculino; si los hombres pueden hacer investigación feminista (teniendo objetivos feministas); la ética de revelar la finalidad de nuestro trabajo o nuestra ideología política a los que simpatizan con nuestras ideas y ocultarlo a los que se negarían a hablar con nosotras si las conocieran; cómo reaccionar ante ideas que nos resultan ofensivas y que los entrevistados expresan con libertad porque les ofrecemos confianza; etc. Quizás lo esencial como geógrafas feministas es ser honestas con nuestra investigación, los dilemas éticos que plantee y los resultados del proceso de investigación (Madge, 1997; McDowell, 2000).

\section{A modo de conclusión}

En definitiva, la metodología y los métodos cuantitativos y cualitativos no son antitéticos, ni el feminismo incita a un elitismo metodológico, sinó que promueve una variedad de ellos. Sin embargo, una in- 
vestigación feminista debe ser consciente de la ambigüedad, complejidad, no-universalidad, del rol de la subjetividad, posicionalidad y reflexividad en el proceso de investigación, y finalmente del compromiso para el cambio social.

En su búsqueda de metodologías y métodos en consonancia con sus objetivos, la geografía feminista (y las geógrafas rurales feministas) han realizado un ejercicio de reevaluación de las metodologías y los métodos, y han desarrollado la metodología cualitativa extendiéndola y legitimándola en toda la investigación geográfica ${ }^{11}$. Además, su contribución a la disciplina va más allá, a través de la reconceptualización, la introducción de nuevas teorías, nuevos temas y nuevos conceptos. Y para el estudio de las mujeres del medio rural esto ha sido muy importante, para visibilizarlas, empoderarlas y diferenciarlas, en distintos tiempos y contextos.

En España se ha recorrido un importante camino en geografía rural y género pero queda mucho por hacer a nivel teórico, empírico y de contribución metodológica. En el marco de una Unión Europea y una política común en desarrollo rural que debe atender a la diversidad de comunidades y paisajes rurales, es imprescindible aportar conocimiento sobre nuestro contexto, y en la construcción de este conocimiento, el género (y desde él, la extensión a otras categorías de análisis como la clase, la etnicidad, sexualidad, religión, cultura o discapacidad) así como una epistemología que legitime las voces de las personas como conocimiento, es fundamental.

A nivel metodológico, se requiere una exploración de «nuevos» métodos (o menos utizados por los geógrafos/as) y una mayor profundización los existentes, que nos abran también a nuevos temas. En este sentido, los «nuevos» métodos pueden ayudar a comprender las múltiples y complejas relaciones de la vida social rural actual y por lo tanto, a producir un conocimiento contextualizado de la realidad. La investigación colaborativa o los grupos de discusión, por ejemplo, pueden detectar cómo los actores sociales reciben, incorporan, discuten o resisten las fuerzas externas inductoras de cambio en las zonas rurales, $\mathrm{y}$

\footnotetext{
${ }^{11}$ Según Janice Monk (2002), las geógrafas feministas fueron pioneras en el perfeccionamiento de las metodologías cualitativas, la evaluación de los valores relativos de los métodos cuantitativos y cualitativos y el impulso dado a la reflexión y el posicionamiento en la investigación. Fruto de su iniciativa fue la creación en el año 2000 del grupo de trabajo sobre métodos cualitativos en la Asociación de Geógrafos Americanos (AAG). 
cómo se construyen los valores colectivos para generar o resistir estos cambios. Los métodos visuales (prensa, televisión, cine, fotografía, pintura, literatura...) pueden dar luz sobre qué elementos y valores se construyen - y en ocasiones se legitiman - los discursos sobre el medio rural; o sobre cómo las experiencias cotidianas de las mujeres y de los hombres rurales estan imbuidas de ideas y representaciones sobre particulares identidades de género asociadas con lo rural. Los análisis de textos pueden ser enormemente útiles para analizar las políticas rurales a distintos niveles (Unión Europea, políticas nacionales, regionales, locales) y detectar cómo el género y otras categorias de división son representados. Las entrevistas en profundidad, mucho más desarrolladas, tienen mucho potencial para detectar las experiencias subjetivas de los hombres y las mujeres acerca de su vida en el medio rural, sus sentimientos de pertenencia o exclusión a la comunidad rural; conocer a «otros» rurales y sus geografías; o comprender cómo lo rural es cada vez más una construcción social y cultural asociada al tiempo y al espacio, y específica a los individuos y los grupos sociales. Finalmente, los sistemas de información geográfica (SIG) constituyen otra interesante fuente metodológica a examinar. Los SIG han sido utilizados por algunas geógrafas feministas (McLafferty \& Barbara Tempalski, 1995; Hanson et al., 1997) y pueden ser otra técnica útil siempre que la visión descontextualizada y descorporeizada de estos sistemas no sea la forma de producción de conocimiento principal (Kwan, 2002). Por su parte, pueden describir y representar un contexto a muchos niveles de detalle y distintas escalas, pueden integrar espacialmente varios tipos de datos (estadísticos, etnográficos, visuales...) a fin de que los contextos y las experiencias de las personas en estos contextos sean expuestos de forma más elaborada (Hanson, 2002). El trabajo de las geógrafas feministas con los SIG es una tarea importante para la geografía actual y también para los trabajos de geografía y género, siempre que se tenga claro que los métodos y datos proporcionados por los SIG pueden ser utilizados en una determinada investigación y no que sean éstos los que determinen qué investigación realizar.

En definitiva, a través de estas páginas hemos pretendido estimular la investigación de la dinámica de la vida rural con otras lentes más sensibles a la complejidad y a la diferencia. Y para ello, el actual momento de debate epistemológico y metodológico es muy sugerente para tratar nuevos temas y explorar otros métodos de investigación en geografía rural.

Estudios Geográficos, LXV, 254, 2004

$$
-22-
$$




\section{BIBLIOGRAFIA}

Albet, A. y GaRCIA RAMON, M. D.: «Reinterpretando el discurso colonial y la historia de la geografía desde una perspectiva de género», en J. Nogué y J. L. Villanova (eds.), España en Marruecos, Lleida, 1999, Milenio, pp. 55-72.

Almas, R. and HaUgen, M.: «Norwegian gender roles in transition. Masculinization hypothesis in the past and in the future», Journal of Rural Studies, 7, $1991 \mathrm{pp} .79-83$.

ANTIPODE: «Discussion and debate: symposium on feminist participatory research», 27, 1995, pp. 71-101.

Baylina, M.: «Metodología cualitativa y estudios de geografía y género», Documents d'Anàlisi Geogràfica, 30, 1997, pp. 123-138.

BenERÍA, L. (ed.): Women and development. The sexual division of labor in rural societies, New York, 1982, Praeger.

BowlBY, S.: «Feminist geography and the changing curriculum», Geography, 77, 1992, pp. 349-360.

BowlBY, S.; LewIS, J.; MCDowell, L. and FoorD, J.: «The geography of gender» en R. Peet and N. Thrift (eds.), New models in geography 2, London, 1989, Unwin and Hyman.

BRANDTH, B.: «Rural masculinity in transition. Gender images in tractor advertisments», Journal of Rural Studies, 11 (2), 1995, pp. 123-133.

BRANDTH, B. and HAUgen, M.: «From lumberjack to business manager: masculinity in the Norwegian forestry press», Journal of Rural Studies, 16, 2000, pp. 343-355.

BRU, J.: «Las movilizaciones medioambientales desde una perspectiva de género: tres casos de estudio en Catalunya, Andalucía y País Vasco», Mientras Tanto, 65, 1996a, pp. 61-82.

__ «Spanish women against industrial waste» en D. Rocheleau et al. (eds.), Feminist political ecology. Global issues and local experiences, London, 1996b, Routledge, pp. 105126.

ButTimer, A.: «Home-reach-journey» in P. Moss (ed.), Placing autobiography in geography, Syracuse, 2001, Syracuse University Press, pp. 22-40.

CAmARERo, L.; SAMPEDRo, R., y Vicente-MAZARIEgos, J. I.: Mujer y ruralidad: el círculo quebrado, Madrid, 1991, Ministerio de Trabajo y Asuntos Sociales e Instituto de la Mujer.

CANADIAN GEOGRAPHER: «Feminism as method», 37, 1993, pp. 48-61.

CARr, M.; Chen, M. and TATE, J.: «Globalization and home-based workers», Feminist Economics, 6 (3), 2000, pp. 123-142.

CLOKE, P.: «Country backwater to virtual village? Rural studies and "The Cultural Turn"», Journal of Rural Studies, 13, 1997, pp. 367-375.

ClOKE, P. and LITTLE, J.: Contested countryside cultures. Otherness, marginalisation and rurality, London, 1997, Routledge.

Cope, M.: «Feminist epistemology in geography», en P. Moss (ed.), Feminist geography in practice: Research and methods, Oxford, 2002, Blackwell, pp. 43-56.

CRANG, M.: «Qualitative methods: the new orthodoxy?», Progress in Human Geography, 26 (5), 2002, pp. 647-655.

Domosh, M.: «Towards a feminist historiography of geography», Transactions of the Institute of British Geographers, 16 (1), 1991, pp. 95-104.

England, K.: «Getting personal: reflexivity, positionality and feminist research», Professional Geographer, 46, 1994, pp. 80-89.

EsIM, S.: «Can feminist methodology reduce power hierarchies in research settings?», $\mathrm{Fe}$ minist Economics, 3, 1997, pp. 137-140.

Eyles, J.: «Been there, done that, what's next?», en P. Moss (ed.), Placing autobiography in geography, Syracuse, 2001, Syracuse University Press, pp. 41-61.

FinCHER, R. and PANELLI, R.: «Making space: women's rural and urban activism and the Australian state», Gender, Place and Culture, 8 (2), 2001, pp. 129-148. 
GARCIA RAMON, M. D.: «Geography and gender in Spain: New lines of research and teaching, Journal of Geography in Higher Education, 13, 1989a, pp. 110-112.

_ : «Actividad agraria y género en España: una aproximación a partir del Censo Agrario de 1982», Documents d'Anàlisi Geogràfica, 14, 1989b, pp. 89-114.

Garcia Ramon, M. D.; Castañer, M. and Centelles, N.: «Women and geography in Spanish universities», Professional Geographer, 40 (3), 1988, pp. 307-315.

Garcia Ramon, M. D.; Cruz, J.; Salamaña, I. y Villarino, M.: Mujer y agricultura en España. Género, trabajo y contexto regional, Vilassar de Mar, 1995, Oikos-tau.

GARCIA RAMON, M. D. y NogUÉ, J.: «Colonialismo, imperialismo y exploración en geografía: nuevas aportaciones críticas sobre orientalismo y postcolonialismo», en J. Nogué y J. L. Villanova (eds.), España en Marruecos, Lleida, 1999, Milenio, pp. 35-54.

GARCIA RAMON, M. D. y BAYLINA, M.: «Estudios rurales y género en Europa y en España: Un estado de la cuestión», en M. D. Garcia Ramon y M. Baylina (eds), El nuevo papel de las mujeres en el medio rural, Vilassar de Mar, 2000, Oikos-tau, pp. 23-64.

_- :Comment on "El nuevo papel de las mujeres en el desarrollo rural" (The new role of women in rural development)», Antipode, (en prensa), 2002.

GIRADAKOU, I.: «Young's women attitudes towards agrciculture and women's new roles in the Greek countryside: a first approach», Journal of Rural Studies, 15 (2), 1999 pp. 147-158.

HALFACREE, K.: «Out of place in the country: travellers and the "rural idyll"», Antipode, 28, 1996, pp. 42-72.

HANSON, S.: «Geography and feminism: worlds in collision», Annals of the Association of American Geographers, 82, 1992, pp. 569-586.

- : «Connections», Gender, Place and Culture, 9 (3), 2002, pp. 301-303.

HANSON, S. and Monk, J.: «On not excluding half of the human in human geography», Professional Geographer, 34, 1982, pp. 11-23.

Hanson, S. and Pratt, G.: «The Worcester Study» en Gender, work and space, London, 1995, Routledge, pp. 54-92.

HANSON, S.; KOMINIAK, T. and CARLIN, S.: «Assessing the impact of location on women's labour market outcomes: a methodological exploration», Geographical Analysis, 29, 1997, pp. 282-297.

HARAWAY, D.: «Situated knowledges: the science question in feminism and the privilege of partial perspective», Feminist Studies, 14, 1988, pp. 575-599.

HARDInG, S. (ed.): Feminism and methodology, Milton Keynes, 1987, Open University Press.

Hughes, A.: «Women and rurality: gendered experiences of "community" en village life» en P. Milbourne (ed.), Revealing rural others: representation, power and identity in the British countryside, London, 1997, Pinter, pp. 167-188.

JoHNSON, L. C.: «The difference feminism makes: Researching unemployed women in an Australian region» en P. Moss (ed.), Feminist geography in practice, London, 2002, Blackwell, pp. 57-74

Jones, J. P.; NAST, H. and RoBerTs, S.: Thresholds in feminist geography, Latham, MD, 1997, Rowman and Littlefield.

KIM, M.: «Poor women survey poor women: Feminist perspectives in survey research», $\mathrm{Fe}$ minist Economics, 3, 1997, pp. 99-118.

KwAN, M. P.: «Is GIS for women? Reflections on the critical discourse in the 1990's», Gender, Place and Culture, 9 (3), 2002, pp. 271-279.

KoBAYASHI, A.: «Colouring the field: gender, "race" and the politics of fieldwork», Professional Geographer, 46, 1994, pp. 73-79.

LiTTLE, J.: «Constructions of rural women's voluntary work», Gender, Place and Culture, 4 (2), 1997, pp. 197-209.

- : «Otherness, representation and the cultural construction of rurality», Progress in Human Geography, 23 (3), 1999, pp. 437-442. 
LiTTLE, J.: Gender and rural geography. Identity, sexuality and power in the countryside, Harlow, 2001, Prentice Hall, pp. 18-45.

- : «ural geography: rural gender identity and the performance of masculinity and femininity in the countryside», Progress in Human Geography, 26 (5), 2002, pp. 665-670.

LÓPEZ GARCíA, R. M.: «La agricultura ecológica en el quehacer científico. Tema incipiente en la geografía», Anales de Geografía de la Universidad Complutense, 19, 1999, pp. 351-364.

- : «La agricultura ecológica como una alternativa para las mujeres», Actas del $X$ Coloquio de Geografía Rural de la Asociación de Geógrafos españoles, Lleida, 2000, AGE and Universitat de Lleida, pp. 490-498.

Lowe, P. et al.: «Regulating the new rural spaces: issues arising from the uneven development of rural land», Journal of Rural Studies, 9, 1993, pp. 205-222.

MCLAFFERTY, S. and TEMPALSKY, B.: «Restructuring and women's reproductive health: implications for low birthweight in New York City, Geoforum, 26, 1995, pp. 309-323.

MADGE, C. et al.: «Methods and methodologies in feminist geographies: politics, practice and power», en Women and Geography Study Group (ed.), Feminist Geographies. Explorations in diversity and difference, Harlow, 1997, Addison Wesley, pp. 86-111.

MAJORAL, R. y SÁNCHEZ, D.: «Trabajo femenino, tamaño y orientación técnico-económica de las explotaciones agrarias en España», Actas del X Coloquio de Geografía Rural de la Asociación de Geógrafos españoles, Lleida, 2000, AGE and Universitat de Lleida, pp. 499-507.

Manning, P.; Denman, C. and Monk, J.: «Seeking common ground: Collaboration in research and action at the Mexico-US border», 2002 (versión multicopiada).

MARCUS, G. E.: «Ethnography in/of the world system: The emergence of multi-sited ethnography», Annual Review Anthropology, 24, 1995, pp. 95-112.

MARSDEN, T.: «Economic perspectives» in B. Ilbery (ed.), The geography of rural change, Harlow, 1998, Longman, pp. 13-30.

- : «Opening the boundaries of the rural experience: progressing critical tensions», Progress in Human Geography, 18 (4), 1994, pp. 523-531.

- «Rural geography trend report: the social and political bases of rural restructuring», Progress in Human Geography, 20 (2), 1996, pp. 246-258.

MATTHEWS, H. et al.: «Growing-up in the countryside: children and the rural idyll», Journal of Rural Studies, 16 (2), 2000, pp. 141-153.

MCDOwELL, L.: «Coming from the dark: feminist research in geography» en J. Eyles (ed.), Research in Human Geography, Oxford, 1988, Blackwell, pp. 155-173.

- - «Doing gender: feminism, feminists and research methods in human geography», Transactions of the Institute of British Geographers, 17, 1992, pp. 399-418.

- : «Women, gender, feminists: doing feminist geography», Journal of Geography in Higher Education, 21, 1997, pp. 381-400.

—.: Género, identidad y lugar, València, 2000, Cátedra.

Momsen, J. H.: «Género y agricultura en Inglaterra», Documents d'Anàlisi Geogràfíca, 14, 1989 , pp. 115-130.

Momsen, J. H. and KINNAIRD, V. (eds.): Different places, different voices. Gender and development in Africa, Asia and Latin America, London, 1993, Routledge.

Monk, J.: «Gender in the landscape. Expressions of power and meaning» en K. Anderson and F. Gay (eds.), Cultural Geographies, London, 1999, Longman, pp. 153-172.

- - «Many roads. The personal and professional lives of women geographers", in P. Moss (ed.), Placing autobiography in geography, Syracuse, 2001, Syracuse University Press, pp. 167-187.

: «Continuidades, cambios y retos de la geografía contemporánea en los Estados Unidos», Documents d'Anàlisi Geogràfica, 39, 2002, pp. 75-95. 
MonK, J. and HANSON, S.: «On not excluding the other half of the human in geography», The Professional Geographer, 34, 1982, pp. 11-23.

Monk, J.; Manning, P. and Denman, C.: «Working toguether: Feminist perspectives on collaborative research and action», 2002, (en prensa, aceptado en ACME: An International E-Journal for Critical Geographies).

Moss, P.: «Engaging autobiography», in P. Moss (ed.), Placing autobiography in geography, Syracuse, 2001, Syracuse University Press, pp. 188-198.

: «Taking on, thinking about, and doing feminist research in geography» en P. Moss (ed.), Feminist geography in practice: Research and methods, Oxford, 2002, Blackwell, pp. 1-17.

Murdoch, J. and PratT, A.: «Modernism, postmodernism and the post-rural», Journal of Rural Studies, 9, 1993, pp. 411-427.

PANELLI, R.: «Young rural lives: strategies beyond diversity», Journal of Rural Studies, 18 (2), 2001, pp. 113-122.

PINI, B.: «Focus groups, feminist research and farm women: opportunities for empowerment in rural social research», Journal of Rural Studies, 18 (3), 2002, pp. 339-351.

Prados, M. J.: Situación socioeconómica de las mujeres rurales en España, Sevilla, 2002, Consejería de Agricultura y Pesca, y Comunidad Europea.

PRATS, M.: «Geografia feminista i metodologia: Reflexió sobre un procés d'aprenentatge paral.lel», Cuadernos de Geografía, 64, 1998, pp. 313-323.

PROFESSIONAL GEOGRAPHER: «Women in the field», 46, 1994, pp. 54-102.

- : :Should women count? The role of quantitative methodology in feminist geographic research», 47, 1995, pp. 426-466.

- : «Women in geography in the 21st Century», 54, 2001, pp. 697-738.

Raghuram, P.; MADGE, C. and Skelton, T.: «Feminist research methodologies and student projects in Geography», Journal of Geography in Higher Education, 22 (1), 1998, pp. $35-48$.

Rose, D.: «Revisiting feminist research methodologies: A working paper», draft submitted to Status of Women Canada, Research Directorate, 2002.

Rowbotham, S. and TATE, J.: «Homeworking: new approaches to an old problem» en E. Drew, R. Emerek and E. Mahon (eds.), Women, work and the family in Europe, London, 1998, Routledge, pp. 112-123.

RURAL SOCIOLOGY: Special issue, 64, 2000.

SABATÉ, A. and Tulla, A.: «The geography of gender: The state of the art» in J. Bosque Maurel et al. (eds.), Geography in Spain (1970-1990), Madrid, 1992, Fundación BBV, pp. 261-267.

SABATÉ, A.: «Geografía y género en el medio rural: Algunas líneas de análisis», Documents d'Anàlisi Geogràfica, 14, 1989, pp. 131-147.

- : «Género, medio ambiente y acción política: un debate pendiente en la geografía actual», Anales de Geografía de la Universidad Complutense, 20, 2000, pp. 177-191.

SABATÉ, A.; RodríGuez, J. M. y DíAz, M. A.: Mujeres, espacio y sociedad. Hacia una geografía del género, Madrid, 1995, Síntesis.

SAmARASINGHE, V.: «Counting women's work: The intersection of time and space» en J.P. Jones; H. Nast and S. Roberts (eds.), Thresholds in feminist geography: difference, methodology and representation, Latham, 1997, MD, Rowman and Littlefield.

SAMPEDRO, R.: Género y ruralidad. Las mujeres ante el reto de la desagrarización, Madrid, 1996, Ministerio de Trabajo y Asuntos Sociales, e Instituto de la Mujer.

SolsonA, M.: «El problema de la medición del trabajo de la mujer»,_Documents d'Anàlisi Geogràfica, 14, 1989, pp. 149-169.

Thorsen, L. E.: «Interpreting farm women's lives: Reflections on the bibliographical method» en L. Van der Plas and M. Fonte (eds.), Rural gender studies in Europe, Assen, 1994, Van Gorcum, pp. 147-153. 
TownsEnd, J.: «Es pot parlar en nom dels altres? Es pot, des de fora, representar les dones pioneres de la selva tropical mexicana?», Documents d'Anàlisi Geogràfica, 26, 1995, pp. 209-218.

__ : «Gènere i canvi agroecològic a l'ocupació de la terra a Mèxic», Documents d'Anàlisi Geogràfica, 35, 1999, pp. 101-117.

Townsend, J.; Arrevillaga, U.; Cancino, S.; Pacheco, S. y PÉrez, E.: Voces femeninas de la selva, Universidad de Durham, México, 1994, Centro de Estudios del Desarrollo Rural.

VAn DAm, F., Heins, S. and Elbersen, B.: «Lay discourses of the rural and stated and revealed preferences for rural living. Some evidence of the existence of a rural idyll in the Netherlands», Journal of Rural Studies, 18 (4), 2002, pp. 461-476.

Vicente-Mazariegos, J. I.; Porto, F.; Camarero, L. y SAmpedro, R.: Situación socioprofesional de la mujer en la agricultura, Madrid, 1991-94, Ministerio de Agricultura, Pesca y Alimentación.

WhatmoRe, S.: «Sustainable rural geographies», Progress in Human Geography, 17 (4), 1993 , pp. 538-547.

_- «Theoretical achievements and challenges in European Rural Gender Studies», in L. Van der Plas and M. Fonte (eds), Rural gender studies in Europe, Assen, 1994, The Netherlands, Van Gorcum, pp. 39-49.

WoMEn AND GEOGRAPHY STUdy GROUP: Geography and gender, London, 1984, Heinemann.

Longman.

WOODWARD, R.: «"It's a man's life!": soldiers, masculinity and the countryside», Gender, Place and Culture, 5, 1998, pp. 277-300.

RESUMEN: El artículo pretende reflexionar sobre la metodología utilizada desde la geografía para la investigación sobre las mujeres y la sociedad rural en Europa y España. En primer lugar se destaca la importancia de la teoría feminista para estas investigaciones, a continuación se analiza la relación entre los temas de estudio, los paradigmas y las metodologías utilizadas en la evolución de los estudios sobre la mujer rural. Finalmente, se valoran las posibilidades, dificultades y la adecuación de los métodos cualitativos y cuantitativos para estas investigaciones.

PALABRAS ClAVE: Metodología, mujeres, género, sociedad rural, Europa, España.

ABSTRACT: The purpose of this article is to reflect on the methodology used from Geography to research on women and rural society in Europe and Spain. First of all, we underline the importance of feminist theory, second, we analize the relationship among the research subjects, paradigms and methodologies used in the evolution of rural women studies, and finally, we assess the possibilities and difficulties of qualitative and quantitative methods to these investigations.

KeY woRDs: Methodology, women, gender, rural society, Europe, Spain.

RÉSUMÉ: L'objectif de cet article est de réflechir sur la méthodologie utilisé d'après la Géographie à propos de la recherche sur l'étude des femmes et la societé rurale en Europe et en Espagne. D'abord, nous soulignons l'importance de la théorie feministe; en second lieu nous analysons la rélation entre les sujets de recherche, les paradigmes et les mét- 
hodologies utilisées dans les études sur les femmes rurales. Et finalement, nous évaluons les possibilities et les difficultés des methodes qualitatives et quantitatives pour ces recherches.

MoTS CLES: Métodologie, femmes, genre, societé rurale, Europe, Espagne.

Estudios Geográficos, LXV, 254, 2004 